\title{
Les maladies des rongeurs domestiques en Guyane française
}

\author{
par H. FLOCH
}

Nous résumons aujourd'hui nos constatations sur les maladies des lapins et des cobayes, en Guyane, d'après nos observations faites de 1939 à 1952 au laboratoire, comme nous avons déjà donné le résumé de nos constatations faites sur les maladies des volailles durant la même période (1) (2).

Les rongeurs domestiques, comme les oiseaux de basse-cour, sont assez difficiles à élever en notre département sud-américain, de climat équatorial.

Les principales affections que nous avons identifiées chez eux, en une quinzaine d'années, sont : la coccidiose et la pasteurellose pour les lapins. la salmonellose pour les cobayes.

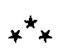

Nous avons isolé et isolons assez fréquemment des pasteurelles de moelle osseuse de lapins atteints d'épizooties rapidement mortelles (pasteurelloses aiguës).

Ces pasteurelles répondent en général aux caractères du germe isolé par nous en 1941 et décrit comme suit (3) :

Petit bacille Gram -, immobile, glucose + , lactose - , maltose - , mannite + , saccharose + , indol + , lyse biliaire - hémolyse -

Nous avons aussi isolé ce microbe, Pasteurella cuniculicida (Koch, 1878) (= P. multocida Lehmann et Neumann, 1899) de clapiers atteints de pasteurelloses subaiguës. C'est ainsi qu'en 1948, nous avons isolé cette pasteurelle de moelles osseuses d'un élevage où 20 lapins étaient malades depuis plusieurs jours : amaigrissement, dyspnée, inappétence, mort. A la même époque d'ailleurs, plusieurs clapiers de Cayenne présentèrent cette même affection contre laquelle, disons le tout de suite, la vaccination par le chromo-vaccin de Remlinger fut très active (deux décès seulement après vaccination dans l'élevage que nous signalons plus haut).

Assez souvent aussi nous avons vu dans les clapiers des cas de pasteurellose chronique se manifestant surtout par du coryza.
Pratiquement tous les lapins en Guyane sont atteints de coccidiose et, ici comme ailleurs, on peut dire que, lorsque des lapereaux de 1 à 3 mois meurent, soit amaigris, soit météorisés, alors que les adultes ne sont pas atteints, 99 fois sur 100, il s'agit de coccidiose, hépatique à Eimeria stiedai (Lindemann, 1865) le plus souvent, mais aussi intestinale à E. magnā (Perard, 1925) et à E. perforans (Leuckart, 1879).

Ces coccidioses, en général, se manifestent par de l'inappétence, de l'amaigrissement, de la polyurie, un «gros ventre » (hépatomégalie ou météorisme) et quelquefois de la diarrhée. la mort survient en quelques jours dans la forme aiguë, en quelques semaines dans les formes subaiguës ou chr oniques.

Lorsqu'un lapin échappe à la première atteinte il possède une sorte d'immunité relative qui permet d'ailleurs en général la propagation de l'affection dans le clapier.

\section{$* *$}

Au point de vue prophylactique et thérapeutique nous insisterons sur la vaccination antipasteurellique.

Nous n'avons eu que peu de succès par la vaccination à l'aide de vaccin tué par la chaleur. Il n'en a pas été de même, en général, à l'aide de la vaccination par le chromo-vaccin de Remlinger (4) (5). Comme nous l'avons dit au sujet des volailles (2), cette vaccination n'est malheureusement pas très pratique en grande série du fait qu'il convient d'injecter aux animaux une sérieuse quantité de vaccin, à plusieurs reprises, à quelques jours d'intervalle. Il n'en est pas moins vrai que nous avons cependant eu d'excellents résultats prophylactiques et même thérapeutiques chez des lapins atteints de pasteurellose subaiguë ou chrunique, et l'on sait combien il est toujours difficile d'immuniser contre les pasteurelles, et plus particulierement les lapins, animaux sensibles a i ces germes par excellence.

Pour obtenir le chromo-vaccin, on ajoute à $20 \mathrm{~cm}^{3}$ 
de culture de pasteurelle en bouillon XX gouttes de solution de fuchsine basique (ou de violet de gentiane) à $1 \%$; vingt-quatre ou vingt-huit heures de contact sont nécessaires pour obtenir la stérilité de la suspension microbienne, stérilité qu'il est bon de vérifier.

Les pasteurelles ont alors perdu leur vitalité mais ont conservé leurs propriétés antigéniques.

Expérimentalement, Remlinger constata qu'il fallait injecter de $28 \mathrm{~cm}^{3}$ à $57 \mathrm{~cm}^{3}$ en injections de $5 \mathrm{~cm}^{3}$ à plusieurs jours d'intervalle, pour obtenir une vaccination effective des aninnaux de basse-cour.

Lorsqu'on utilise des cultures sur gélose, on peut vacciner les lapins à l'aide de $10 \mathrm{~cm}^{3}$ de vaccin mais seulement d'ailleurs lorsqu'on fuchsine 'une suspension microbienne finale riche en pasteurelles, bien plus que ne le sont les cultures en bouillon nutritif. Ici encore d'ailleurs les injections fractionnées sont plus actives et recommandées.

\section{$*^{*} *$}

Au point de vue coccidiose, nous avons obtenu d'excellents résuitats à l'aide de sulfamidés, notamment de Sulfaméthyldiazine et de Sulfaquinoxaline (plus toxique mais moins coûteuse). Il convient de traiter preventivement les jeunes lapins, surloul au moment du sevrage, et d'appliquer le traitement évidemment aussi en cas de mortalité due à la coccidiose.

En effet ces sulfamidés et quelques autres : Sulfaguanidine, Sulfapyridine, Sulfapyrazine ont surtout une action préventive lorsqu'ils sont additionnés au taux de $1 \%$ dans la nourriture. On les utilise aussi en solution dans de l'eau, quand celd esl possible, à raison de $\mathrm{l} g$ de corps actif par litre.

On peut considérer ces sulfamides comme de véritables 《coccidiostatiques »; leur action est liee à la présence d'un groupement amine libre en position para a11 noyalı benzénique.

I.es sels de sodium de la Sulfaméthazine et de la Sulfapyrazine sont plus actifs et ont même une action curative.

Il ne faut pas utiliser les sulfamides pendant plus d'une semaine de façon continue car on pourrait alors amener l'apparition de symptômes d'avitaminoses dans le clapier.

La Quinacrine et la Nivaquine (1 cg par kilogramme de poids d'animal pendant six jours) donnent aussi de bons résultats dans le traitement de la coccidiose des lapins : l'aflection aiguë ou subaiguë disparaît pour faire place à une affection chronique bénigne.

Il est de même de la Phénothiazine, anthelminthique très actif, notamment contre les nématodes des volailles (2), utilisée pendant six jours à la dose quotidienne de $0,5 \mathrm{~g}$ à $\mathrm{I} \mathrm{g}$ par kilogramme de poids.

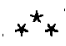

Chez le cobaye nous avons isolé (mais rarement seulement) des pasteurelles (par exemple en 1947).

Il s'agissait de Pasteurella caviseptica (Nicolle et Refick bey, 1896) très voisine au moins de $P$. avicida (Pasieur 1880) $=$ P. multocida (Lehmann et Neumann, 1899).

Cette pasteurelle provoque une septicémie hémorragique du cobaye avec inflammation fibrino-purulente des séreuses et la " maladie du nez ». Elle est différente de P. caviae (Toni et Trévisan, 1889) agent d'une épizootie à manifestations pseudo-tuberculeuses, et de P. heymanril (Heymann et Kyriasides, 1922) causant des épidémies très meuririères chez le cobaye (péritonite séro-purulente, congestion du foie, de la rate, des surrénales, sans hémorragies). Elle serait différente aussi de P. pericarditis (Roth, 1934) agent d'une pleuro-péricardite fibrineuse (6).

La plupart du temps, en cas d'épizootie, et pratiquement chaque anrée, nous obtenons par ensemencement des moelles osseuses de cobaye, des salmonelles identifiées à Salmonella typhi-murium (Loeffler, 1892).

Voici que nous écrivions dans un rapport annuel à ce sujet (7):

Fn 1945, dans notre élevage de cobayes, nous avons isolé un bacille mobile, Gram --, agglutiné par un sérum stock anti-salmonelle et dont les caractères biochirniques correspondaient à ceux de S. typhi-murium : glucose + , gaz, maltose $t$, mannitol + , lactose - , sucrose - , xylose + , rhamnose + , arabinose + , lait alcalinisé, réaction de V.P. -, réaction du M.R. - , indol -.

Cette identification a été confirmée sérologiquement par Miss Coleman et J.-M. Coffrey a qui nous avons fait parvenir des cultures et que nous remer cions bien sincèrement.

S. typhi-murium cause une maladie naturelle mortelle hautement contagieuse des rats et des souris. Cette salmonelle a été signalée à l'origine de graves épidémies dans des élevages de laboratoire de cobayes sous le nom de B. pestis caviae, (Wherry, 1908).

Quoique S. typhi-murium s'attaque de préférence aux rongeurs, elle a été aussi isolée de perroquets, B. psittacosis (Nocard, 1893) et surtout de canards, de poulets, de pintades, de dindons, de faisans.

Au sujet des salmonelles des volailles (2) nous avons signalé l'importance de S. typhi-murium dans 
les intoxications alimentaires, $B$. aertricke (Nobele, 1899 ) et $B$. enteritidis (Januschke, 1924); nous n'y reviendrons pas ici.

Le vaccin tué par la chaleur ne nous a pas donné de résultats aussi rapides et aussi favorables dans la salmonellose des cobayes que ceux que nous attendions de son emploi en raison des constatations que nous avions faites dans la salmonellose des volailles après usage de vaccin tué par la chaleur (1). Institut Pasteur de la Guyane française.

\section{BIBLIOGRAPHIE}

(1) FLOCH (H.). - Sur la pathologie vétérinaire en Guyane française. Les affections des volailles (I). Salmonelloses. Publication $n^{\circ} 293$ de l'I.P. de la Guyane, août 1953.

(2) FLOCH ( $\mathrm{H}$.). - - Sur la pathologie vétérinaire en Guyane française. Les affections des volailles (II). Pasteurellose, coccidiose, variolo-diphtérie, syngamose, spirochétose, parasitismes divers, tumeurs. Publication no 295 de l'I.P. de la Guyane, septembre 1953.

(3) FLOCH (H.). - Rapport sur le fonctionnement technique de l'Institut Pasteur de la Guyane pendant l'année 1941. Publication no 34 de l'I.P. de la Guyane, mars 1942.

(4) REMLINGER (P.). - Chromo-vaccination des pasteurelloses. Archives de l'I.P. d'Algérie. T. XXIII, no 1, 1945, p. 50.

(5) RFMLINGER (P.). - Chromo-vaccination de la poule contre le choléra. Archives de l'I.P. d'Algerie. T. XXIII, nº 3, I945, p. 176.

(6) HAUDUROY (P.). - Dictionnaire des bactéxies pathogènes poux l'homme, les animaux et les plantes. Paris, février 1953.

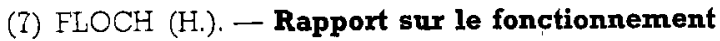
technique de l'Institut Pasteur de la Guyane pendant l'année 1945. Publication $n^{\circ} 125$ de III. de la Guyane, avril 1946. 